\title{
Development of water quality map for Ogbomoso metropolis
}

\begin{abstract}
The quality of water is usually strongly considered as criteria for its suitability for a purpose. Important physical, chemical and bacteriological properties form the basis to judge the quality of water. To determine whether a particular water sample is of acceptable quality for drinking, it is necessary to compare its properties with the regulatory limits of certain well known and accepted standards set by organizations like World Health Organization, US Environmental Protection Agency, Nigeria International Standards etc. The focus of this project is to investigate the extent of contaminants in the available water sources and the causative agents in Ogbomoso North and South Metropolis. 100 water samples were collected through an evenly spread sample point positioning system across the study area, to give an averagely accurate analysis of the result. The Physical properties like Temperature, Turbidity, Total Dissolved Solids; Chemical parameters like Heavy metal concentration and Bacteriological parameters like E-Coli and T-Coli coli form count were analysed and the results were compared with regulatory limits. From the results derived, the physical parameters of tested water samples fall within the WHO standard limits.
\end{abstract}

However, water samples in Ogbomoso North and South Local Government Area contain E-Coli and T-Coli concentration that exceeds the WHO standard of 0 MPN/ $100 \mathrm{ml}$. Wards like Ilogbo (Papa Abede) and Ilogbo/Arada were found to contain high concentration of Iron that exceeds the acceptable $1 \mathrm{mg} / 1$ limit due to the presence of a number of dumpsites and light industries. Phosphate concentration in all water samples tested exceeded the WHO and NIS standard of $5 \mathrm{mg} / 1$, which is as a result of uncontrolled septic waste and fertilizer run-off.

The concentration of Manganese in all water samples across the study area also exceeded the $0.2 \mathrm{mg} / 1 \mathrm{WHO}$ and NIS standard for potable water as a result of the unchecked Industrial activities and vehicle emission in the area. Other areas with significant pollution cases especially Sulphate concentration that exceeds the WHO regulatory limit of $250 \mathrm{mg} / 1$ in water samples include Osupa and Jagun, this can be attributed to the number of the dumping sites present and action of leachates in the areas. The resulting health implication of these findings was discussed. A water quality map was developed to show the varying concentration of water properties across Ogbomoso North and South Local Government Area. The study suggests waste control activities be adopted in the study area and public enlightenment schemes to protect water sources in the area, as the task of reducing the extent of contaminants in water sources in Ogbomoso North and South LGA requires the effort of the residents, government and relative organizations.

Keywords: potable water, contaminants, ogbomoso, north, south
Volume 2 Issue $3-2017$

\begin{abstract}
Ibironke OO,Adegoke OJ,Akindipe RDPark Department of Civil Engineering, Ladoke Akintola University of Technology, Nigeria
\end{abstract}

\author{
Correspondence: Ibironke OO, Department of Civil \\ Engineering, Ladoke Akintola University of Technology, P.M.B \\ 4000, Ogbomoso, Oyo State, Nigeria,
}

Email ibironkeoluwatobi@gmail.com

Received: October 17,2016 | Published: June 06, 2017

\section{Introduction}

Water is a vital resource in the ecosystem since it supports life of all living organisms. Though, it occupies about $70 \%$ of the earth's surface, yet a greater percentage of the world's population, most especially in developing countries live without access to safe water. ${ }^{1}$ This is due to lack of infrastructure for the treatment of water and its eventual distribution for the populace. However, limited access to clean and safe water associated with poor water supply, hygiene and sanitation at household level is widening the poverty gap, gender inequalities and the prevalence of water borne diseases (Gender and Water Alliance (GWA), 2006). This has contributed 3.7\% of the total global disease burden and 2.2million death each year with women and children in the developing countries being the most affected (World Health Organization (WHO)/United Nations International Children's Emergency Fund (UNICEF), 2008). Although the Millennium
Development goals (MDGs) predicted that 'by 2015 the proportion of people without access to safe drinking water and sanitation be very low $^{2}$ " it is anticipated that Africa will only reach the MDGs water target by 2040. 400 million of the people living in Africa will be left without access to safe water with a majority of them being women and children that lives in both rural and urban areas. ${ }^{3}$ Women are the most vulnerable because in most societies, it is women's responsibility to ensure that there is enough clean and safe water for their households for domestic usage and other purposes. ${ }^{4-7}$ The World Health Organization (WHO) estimated that more than $20 \%$ of the world population (around 1.3billion people) have no safe drinking water and that more $40 \%$ of all populations lack adequate sanitation. People in the rural areas in absence of potable water are exposed to the risk of water borne diseases like cholera, typhoid and other environmental implications. There is evidence of widespread contamination of water 
resources in many areas of Nigeria. It should however be noted that not all contamination events pose a threat to our health. Anchored on various records of water-related health crises being noted in the Ogbomoso South Local Government (OSLG) Area and Ogbomoso North Local Government (ONLG), this research work focuses on the investigation of the contamination levels of some selected wells in the area and studying their associated health implications on human beings. With the present death of information on such occurrences in the study area, a study of this nature is expected to provide technical explanations on the causes and implications of these incidents of water-borne diseases on human health. The OSLG has a population of 100,815 people and ONLG has the city's largest population with a figure of 235,710 residents. With increase in agricultural activities and domestic chores in the area, there is greater need for the adequate supply of good quality water for the growing population. As the well water is naturally provided to every being, and then the environmental and health implications that were attached to this natural resource cannot be over-emphasized. Therefore, this project is necessary for the assessment and improvement of the quality of water sources in Ogbomoso.

\section{The study areas}

Ogbomoso Township is constituted by the North and South local government councils; it is located in the western part of Oyo state of Nigeria. Ogbomoso North Local Government Area came into existence on September 24, 1991, as a result of the splitting of the former Ogbomoso metropolis into two on Tuesday. Ogbomoso North Local Government Area is bounded in the North and the East by Surulere Local Government Area, in the South by Ogbomoso South Local Government Area and in the West by Orire Local Government Area. Ogbomoso North has its headquarters located at Kinnira, Ogbomoso and Ogbomoso North is urban in outlook. ${ }^{8}$ The major occupations of the population are farming, trading, teaching and artisans. The local government is largely populated by the Yoruba but various other tribes are found in their thousands. Most residents of the Ogbomoso metropolis are either Christians or Muslims; a few others freely worship traditional gods. The local government council is constituted by Ten (10) wards and they are: Masifa/Aguodo, Sabo/ Taara, Isale-Afon, Okelerin, Osupa, Aaje/Ogunbado, Jagun, ItaAlasa, Isale-Ora/Saja and Abogunde. Ogbomoso south LGA occupies an area land mass of about 30 square kilometres and is bounded in the north by Ogbomoso North Local Government, in the south by OgoOluwa Local Government, in the east by Surulere Local Government and in the west by Orire Local Government. Ogbomoso South has its headquarters located at Sunsun/Arowomole, Ogbomoso. It's being in the savannah zone makes farming the major occupation of the people of the area. Other occupation they engage in are trading, teaching and artisans. The Ten (10) wards of the local government are: Ibapon, Ijeru I, Ijeru II, Arowomole, Akata, Alapata,Isoko, Ilogbo, Lagbedu/ Isapa and Oke-Ola/Farm settlement ${ }^{8}$ (Figure 1).

\section{Water pollution in Ogbomoso North and South Local Government (ONSLG)}

Waste accumulating in the study area was found to be produced from various sources. These include:

i. Domestic waste from households,

ii. Refuse from commercials offices and business holdings, iii. Refuse from the market,

iv. Animal remains and dung from the abattoir

v. Trash swept from all kinds of streets including highways arterial and sub arterial roads, e.g. Residue from all types of sanitary facilities in the form of human excreta, toilet papers and the likes.

vi. Waste from the hospitals,

vii. Refuse from Community holdings like Schools, Churches, Clubs, mosque etc.

viii. Dry animal excreta (cows, chickens e t c).

At present, uncontrolled dumping of collected animal excreta in low lying land and canal located in Ogbomoso area is going on. The area is full of dumped solid waste which leads to environmental pollution. The means by which the residents get rid of these wastes is thorough burning and probably if rains fall, sweeps them from location which also leads to the accumulation of the wastes at the drainage system. These could also lead to the run off sweeping away shops and houses and through the blockage of the normal drainage and these could cause a severe loss in lives and properties. ${ }^{9}$

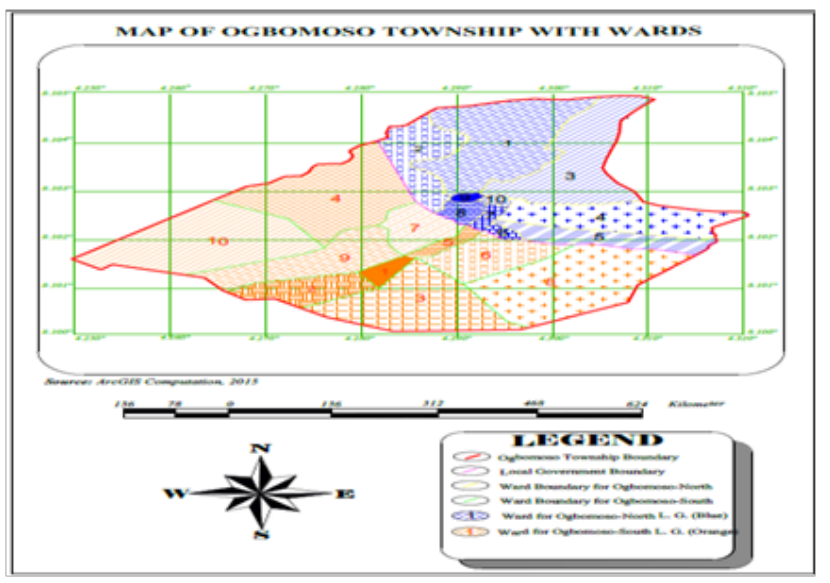

Figure I Map of Ogbomoso North and South with constituent wards.

\section{Water Pollutants and their effects on human health}

Water pollution has become worldwide concern for the past few decades. It is well known that some heavy metals are harmful and cause tonic effects to human beings. The waste discharged by industries, contain compounds of metals, organic compounds, alkalis, phenols etc, which make water to be polluted. Sewage is the dirty which contains human and animal excretion (urine and faeces). This is produced everyday by human activities like bathing, washing of clothes, kitchen water waste discharge, excreting urine and faeces. Solid waste comprises all the waste arising from human and animal activities. According to World Health Organization (WHO); solid waste is regarded as useless, unwanted or discarded materials arising from domestic, trade, commercial, industrial and agricultural as well as from public services. Some petroleum chemicals can also cause some changes in the behaviour and the physical composition of waste component varies widely with location and season of the year. ${ }^{9}$ The resulting health implications of the existence of these waste pollutants are shown in Tables 1-3. 
Table I Physical/organoleptic parameters

\begin{tabular}{llll}
\hline Colour & TCU & $\mathbf{1 5}$ & None \\
\hline Odour & - & Unobjectionable & None \\
Taste & - & Unobjectionable & None \\
Temperature & Celsius & Ambient & None \\
Turbidity & NTU & 5 & None \\
\hline
\end{tabular}

Source: (Nigerian standard for drinking water quality, 2007).

Table 2 Inorganic constituents

\begin{tabular}{|c|c|c|c|}
\hline Parameter & Unit & Max permitted & Health impact \\
\hline Aluminum (Al) & $\mathrm{mg} / \mathrm{l}$ & 0.2 & Potential Neuro-degenerative disorder \\
\hline Arsenic (As) mg/l & $\mathrm{mg} / \mathrm{l}$ & 0.01 & Cancer \\
\hline Barium & $\mathrm{mg} / \mathrm{l}$ & 0.7 & Hypertension \\
\hline Cadmium $(\mathrm{Cd})$ & $\mathrm{mg} / 1$ & 0.003 & Toxic to the kidney \\
\hline Chloride $(\mathrm{Cl})$ & & 250 & None \\
\hline Chromium & $\mathrm{mg} / \mathrm{l}$ & 0.05 & Cancer \\
\hline Conductivity & $\mathrm{Us} / \mathrm{cm}$ & 1000 & None \\
\hline Copper & $\mathrm{mg} / \mathrm{l}$ & 1 & Gastrointestinal disorder \\
\hline Cyanide (CN-) & $\mathrm{mg} / \mathrm{l}$ & 0.01 & Very toxic to the nervous system \\
\hline Fluoride & $\mathrm{mg} / 1$ & 1.5 & Fluorosis, Skeletal tissue morbidity \\
\hline Hardness & $\mathrm{mg} / 1$ & 150 & None \\
\hline Iron & $\mathrm{mg} / 1$ & 0.3 & None \\
\hline Lead $(\mathrm{Pb})$ & $\mathrm{mg} / 1$ & 0.01 & Cancer, affect mental development \\
\hline Magnesium & $\mathrm{mg} / \mathrm{l}$ & 0.2 & Consumer acceptable \\
\hline Manganese & $\mathrm{mg} / 1$ & 0.2 & Neurological disorder \\
\hline Mercury (Hg) & $\mathrm{mg} / \mathrm{l}$ & 0.001 & Affects the kidney \\
\hline Nickel (Ni) & $\mathrm{mg} / 1$ & 0.02 & Possible carcinogenic \\
\hline Nitrate & $\mathrm{mg} / 1$ & 50 & Cyanosis and asphyxia in infants \\
\hline Nitrite & $\mathrm{mg} / 1$ & 0.2 & Cyanosis and asphyxia in infants \\
\hline $\mathrm{pH}$ & - & $6.5-8.5$ & None \\
\hline Sodium $(\mathrm{Na})$ & $\mathrm{mg} / 1$ & 200 & None \\
\hline Sulphate & $\mathrm{mg} / \mathrm{l}$ & 100 & None \\
\hline TDS & $\mathrm{mg} / \mathrm{l}$ & 500 & None \\
\hline Zinc (Zn) & $\mathrm{mg} / 1$ & 3 & None \\
\hline
\end{tabular}

Source: (Nigerian standard for drinking water quality, 2007).

Table 3 Microbiological limits

\begin{tabular}{llll}
\hline Parameter & Unit & Max permitted Level & Health impact \\
\hline $\begin{array}{l}\text { Total Coliform } \\
\text { count }\end{array}$ & $\mathrm{cfu} / \mathrm{ml} 10$ & $\begin{array}{l}\text { Indication of fecal } \\
\text { contamination }\end{array}$ & $\begin{array}{l}\text { Thermo tolerant } \\
\text { Urinary tract infections, bacteraemia, meaningtis, diarrhea (one of the main } \\
\text { causes of morbidity and mortality among children), acute renal failure and } \\
\text { haemolyticanaemia }\end{array}$ \\
$\begin{array}{llll}\text { Faecal } \\
\text { Contamination }\end{array}$ & $\mathrm{cfu} / 100 \mathrm{ml}$ & 0 & Indication of recent faecal Streptococcus \\
\hline
\end{tabular}

Source: (Nigerian standard for drinking water quality, 2007).

Citation: Ibironke OO,Adegoke OJ,Akindipe RD. Development of water quality map for Ogbomoso metropolis. MOJ Eco Environ Sci. 20I7;2(3): I2I-I34. DOI: 10.15406/mojes.2017.02.00025 


\section{Materials and methods}

In locating sampling points for the purpose of this project, boreholes, wells and rivers were identified across Ogbomoso, from which water samples were collected. Ogbomoso North and South LGA consists of 10 wards each, five samples were taken from each ward as shown in Figure 1. Thus, a total of 100 water samples were collected. Prior to the collection of water samples from each water source, a thorough familiarization process was embarked on, so as to give advanced knowledge of the location and course of the sample source as well as references to map using Global Positioning Satellite (GPS) for locating coordinates and referencing on map. Figure 2 shows the sample points located on a map of Ogbomoso North and South LGA.

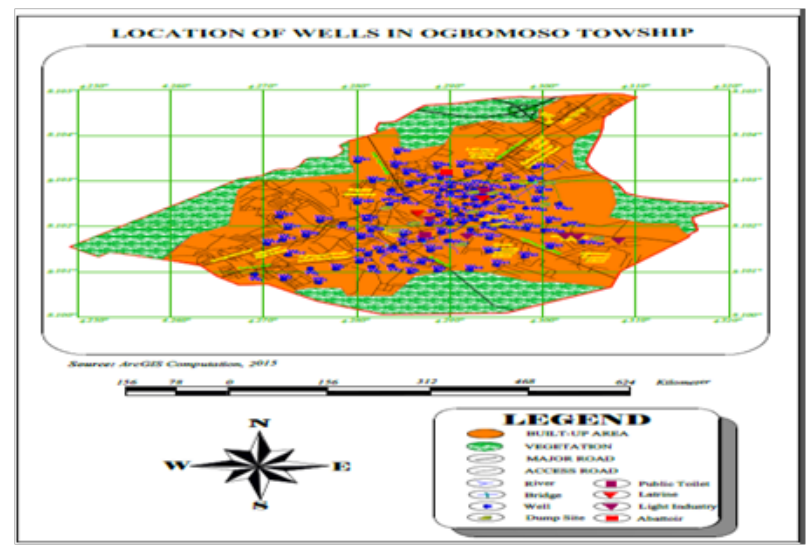

Figure 2 Ogbomoso North and South LGA Map showing location of samples.

\section{Collection of water samples}

Samples were taken at several sources within the study area, in order to determine the quality and pollution state of the water source. The samples were collected into acid pre-cleaned highdensity $1 \mathrm{~L}$ polyethylene sampling bottle in order to prevent the risk of contamination and a possibility of influencing laboratory test results. Fifty centilitre $(50 \mathrm{cl})$ of water samples was collected from each point using a water sampler. The samples were collected early in the morning to avoid disturbances in the properties like increased temperature as a result of human activities etc. The temperature of the samples was tested immediately using a thermometer. Measurements like salinity, bacteriological properties, and dissolved oxygen could not be determined on site due to unavailability of instrument. Hence, the reason for laboratory tests of each sample. The samples were taken to the water and sanitation laboratory for further analysis. ${ }^{10-13}$

\section{The parameters analysed for}

Physical parameters: turbidity, pH value, temperature.

Chemical \& Heavy parameters: Sulphate, Phosphate, Calcium hardness, Magnesium hardness, Manganese, Total hardness, Total dissolved solids, Suspended solids, Lead, Mercury, Chromium, Cadmium, Iron, Zinc, Nickel, Copper, Chloride.

Bacteriological parameters: E- Coli, Total coliform.

\section{Results and discussion}

The physical, chemical and bacteriological qualities of collected water samples in Ogbomoso North and South local governments areas are presented in Tables 4-6 respectively.

Table 4 Chemical quality of water samples in Ogbomoso North and South Local Government of Oyo State

\begin{tabular}{lllllllll}
\hline $\mathbf{S} / \mathbf{N}$ & $\begin{array}{l}\text { Lead } \\
\mathbf{m g} / \mathbf{l}\end{array}$ & $\begin{array}{l}\text { Manganese } \\
\mathbf{m g} / \mathbf{l}\end{array}$ & iron mg/l & Cadmium mg/l & Copper mg/l & Zinc mg/l & Nickel mg/l & Chromium mg/l \\
\hline 1 & 0.01 & 3.25 & 0.01 & 0.01 & 0.1 & 0.09 & 0.14 & 0.049 \\
2 & 0 & 3.18 & 0 & 0.02 & 0.09 & 0.05 & 0.06 & 0.063 \\
3 & 0 & 1.1 & 0 & 0.01 & 0.08 & 0.06 & 0.04 & 0.101 \\
4 & 0 & 2.71 & 0.01 & 0.02 & 0.07 & 0.04 & 0.03 & 0.135 \\
5 & 0.06 & 4.11 & 0 & 0.01 & 0.07 & 0.08 & 0.05 & 0.152 \\
6 & 0.04 & 4.21 & 0 & 0.02 & 0.09 & 0.15 & 0.03 & 0.123 \\
7 & 0.09 & 3.05 & 0 & 0.04 & 0.07 & 0.06 & 0.02 & 0.143 \\
8 & 0 & 3.2 & 0 & 0.04 & 0.07 & 0.21 & 0.12 & 0.154 \\
9 & 0.03 & 3.69 & 0 & 0.05 & 0.06 & 0.09 & 0.15 & 0.167 \\
10 & 0.02 & 3.16 & 0 & 0.03 & 0.07 & 0.05 & 0.04 & 0.152 \\
11 & 0.04 & 3.9 & 0 & 0.01 & 0.06 & 0.03 & 0.04 & 0.163 \\
12 & 0.05 & 2.02 & 0 & 0.02 & 0.06 & 0.02 & 0.03 & 0.143 \\
13 & 0.08 & 4.21 & 0.03 & 0.03 & 0 & 0.12 & 0.08 & 0.156 \\
14 & 0 & 3.65 & 0 & 0.01 & 0 & 0.08 & 0.1 & 0.167 \\
15 & 0.03 & 3.17 & 0 & 0.01 & 0 & 0.04 & 0.08 & 0.168 \\
16 & 0.05 & 3.58 & 0.01 & 0.05 & 0.13 & 0.07 & \\
\hline
\end{tabular}


Table Continued

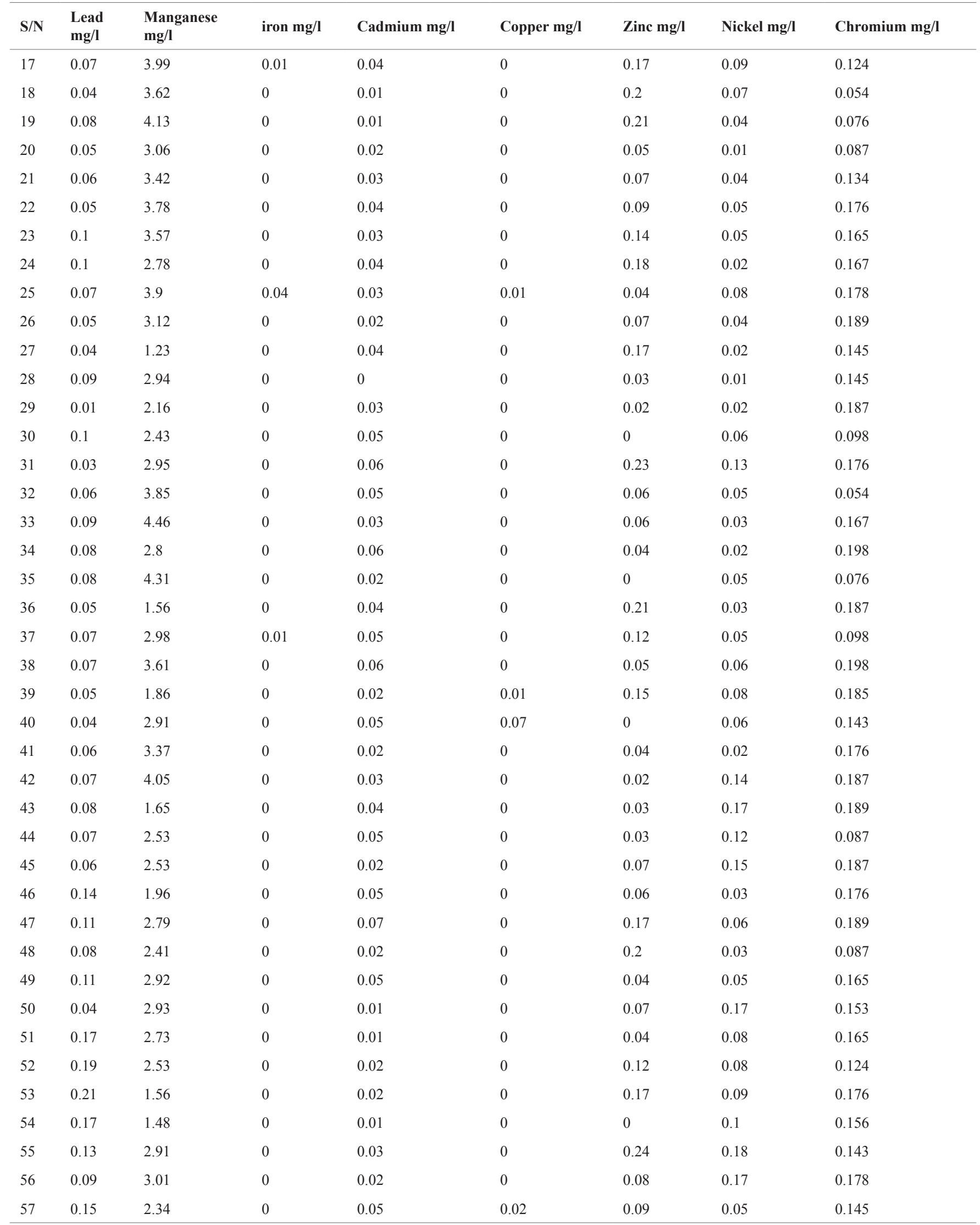


Table Continued

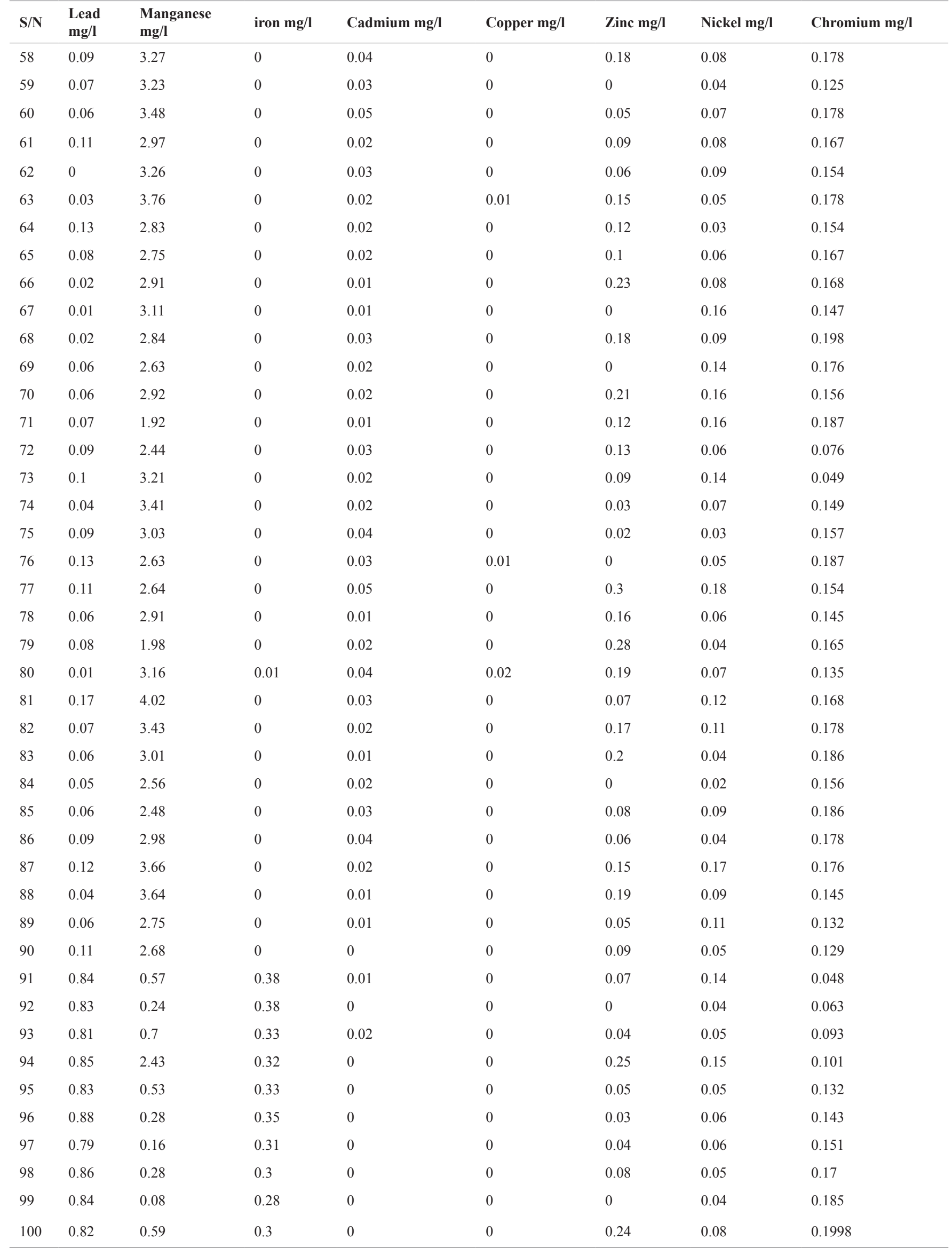


Table 5 Physio-chemical quality of water samples in Ogbomoso North local government of Oyo State

\begin{tabular}{|c|c|c|c|c|c|c|c|c|}
\hline $\mathbf{S} / \mathbf{N}$ & Chloride (mg/l) & Calcium (mg/l) & $\mathrm{Mg}^{2+}(\mathrm{mg} / \mathrm{l})$ & $\begin{array}{l}\text { Total } \\
\text { hardness } \\
(\mathrm{mg} / \mathrm{l})\end{array}$ & Sulphate (mg/l) & $\mathrm{PO}_{4}^{2+}(\mathrm{mg} / \mathrm{l})$ & Temp. $\left({ }^{\circ} \mathrm{C}\right)$ & pH \\
\hline 1 & 2.4 & 12.9 & 12.5 & 25.4 & 370.31 & 10.36 & 31.9 & 5.7 \\
\hline 2 & 4 & 7.9 & 7.6 & 15.5 & 554.98 & 10.37 & 32.1 & 6.9 \\
\hline 3 & 2.2 & 4.2 & 3.9 & 8.1 & 604.29 & 10.37 & 32 & 6 \\
\hline 5 & 10.8 & 9.7 & 7.8 & 17.5 & 439.34 & 10.36 & 32 & 6.6 \\
\hline 6 & 3.1 & 11.5 & 10.1 & 21.6 & 549.6 & 10.36 & 32.1 & 6.6 \\
\hline 7 & 10.8 & 4.2 & 3.3 & 7.2 & 537.05 & 10.48 & 31.8 & 7.2 \\
\hline 8 & 8.2 & 11.3 & 5.9 & 17.2 & 537.06 & 10.36 & 32 & 6.7 \\
\hline 9 & 3 & 8.7 & 7.6 & 16.3 & 103.15 & 10.33 & 32 & 6.6 \\
\hline 11 & 10.4 & 14.7 & 11.3 & 26 & 129.15 & 10.48 & 32.8 & 6.3 \\
\hline 12 & 3.4 & 10.3 & 8.8 & 19.1 & 151.56 & 10.38 & 32 & 6.6 \\
\hline 13 & 9.2 & 7.3 & 5.3 & 12.6 & 268.11 & 10.37 & 32.2 & 6.7 \\
\hline 14 & 9.5 & 7 & 4.9 & 11.9 & 275.28 & 10.38 & 32.2 & 5.5 \\
\hline 15 & 7.7 & 14.2 & 11.6 & 25.8 & 351.48 & 10.39 & 32.9 & 6.4 \\
\hline 16 & 5.6 & 7.1 & 5.6 & 12.7 & 143.49 & 10.36 & 32.8 & 6.6 \\
\hline 17 & 6.2 & 11.9 & 11.1 & 23 & 496.71 & 10.33 & 32.6 & 6.6 \\
\hline 18 & 6.2 & 4.8 & 4.09 & 8.89 & 168.6 & 10.46 & 32.3 & 6.6 \\
\hline 19 & 10.9 & 11.4 & 9.81 & 21.21 & 308.45 & 10.36 & 33 & 7.3 \\
\hline 20 & 11.9 & 10.4 & 8.3 & 18.7 & 306.66 & 10.33 & 33 & 7.3 \\
\hline 22 & 9.9 & 6.2 & 4.5 & 10.7 & 238.52 & 10.48 & 32 & 6.8 \\
\hline 23 & 9.4 & 10 & 9.3 & 19.3 & 165.01 & 10.35 & 32.6 & 6.9 \\
\hline 24 & 11.8 & 6.9 & 6 & 12.9 & 300.38 & 10.5 & 32 & 6.9 \\
\hline 25 & 11.9 & 6.6 & 6.09 & 12.69 & 271.69 & 10.51 & 32.6 & 7.1 \\
\hline 26 & 5.3 & 3.3 & 3.01 & 6.31 & 198.18 & 10.38 & 32.1 & 6.6 \\
\hline 27 & 6.4 & 2.8 & 2.4 & 5.2 & 278.86 & 10.43 & 32.5 & 6.5 \\
\hline 28 & 6.1 & 3.5 & 2.6 & 6.1 & 258.25 & 10.52 & 32.7 & 6.6 \\
\hline 29 & 4.2 & 3.1 & 2.8 & 5.9 & 470.71 & 10.51 & 32.5 & 7 \\
\hline 30 & 8.3 & 5.5 & 4.9 & 10.4 & 469.82 & 10.39 & 32 & 6.4 \\
\hline 31 & 12 & 5.2 & 4.8 & 10 & 166.8 & 10.39 & 32.4 & 6.5 \\
\hline 32 & 13.2 & 5.7 & 5.2 & 10.9 & 253.76 & 10.37 & 32 & 6.4 \\
\hline 33 & 9.2 & 7.2 & 6.8 & 14 & 263.62 & 10.48 & 32.2 & 6.1 \\
\hline 34 & 8.9 & 10.7 & 10.3 & 21 & 253.76 & 10.5 & 32.1 & 6.6 \\
\hline 35 & 9.5 & 4.8 & 4.5 & 9.3 & 284.24 & 10.51 & 32 & 6.7 \\
\hline 36 & 8.9 & 3 & 2.8 & 5.8 & 271.69 & 10.52 & 32.3 & 6.4 \\
\hline 37 & 8 & 3.3 & 3.1 & 6.4 & 142.6 & 10.51 & 32.4 & 6.5 \\
\hline 38 & 7.2 & 5.6 & 5.2 & 10.8 & 447.41 & 10.31 & 32 & 5.9 \\
\hline 39 & 7.7 & 7.9 & 7.3 & 15.2 & 302.17 & 10.512 & 32.2 & 5.9 \\
\hline
\end{tabular}


Table Continued

\begin{tabular}{|c|c|c|c|c|c|c|c|c|}
\hline $\mathbf{S} / \mathbf{N}$ & Chloride (mg/l) & Calcium (mg/l) & $\mathrm{Mg}^{2+}(\mathrm{mg} / \mathrm{l})$ & $\begin{array}{l}\text { Total } \\
\text { hardness } \\
(\mathrm{mg} / \mathrm{l})\end{array}$ & Sulphate (mg/l) & $\mathrm{PO}_{4}^{2+}(\mathrm{mg} / \mathrm{l})$ & Temp. $\left({ }^{\circ} \mathrm{C}\right)$ & pH \\
\hline 41 & 6 & 11.5 & 11 & 22.5 & 469.82 & 10.5 & 32.1 & 6.3 \\
\hline 42 & 8.9 & 3 & 2.8 & 5.8 & 199.97 & 10.32 & 32.6 & 7 \\
\hline 43 & 8.9 & 4.1 & 3.9 & 8 & 301.28 & 10.33 & 32.7 & 5.9 \\
\hline 44 & 5.7 & 8.2 & 5.1 & 13.3 & 300.38 & 10.32 & 32.8 & 6.5 \\
\hline 45 & 10.3 & 8.3 & 8.2 & 16.5 & 292.31 & 10.51 & 33 & 5.5 \\
\hline 46 & 9.1 & 9.9 & 9.2 & 19.1 & 273.49 & 10.32 & 32.1 & 6.9 \\
\hline 47 & 8 & 6.3 & 6.1 & 12.4 & 299.48 & 10.33 & 32 & 7 \\
\hline 48 & 7 & 8.3 & 7.9 & 16.2 & 614.15 & 10.36 & 32.3 & 6.9 \\
\hline 49 & 8.2 & 6.1 & 5.8 & 11.9 & 606.08 & 10.32 & 32.7 & 5.4 \\
\hline 51 & 7.4 & 5.5 & 5.2 & 10.7 & 586.36 & 10.36 & 32.2 & 6.7 \\
\hline 52 & 8.9 & 2.8 & 2.7 & 5.5 & 312.93 & 10.35 & 32 & 6.1 \\
\hline 53 & 9.5 & 2.3 & 2.2 & 4.5 & 411.55 & 10.33 & 32.8 & 6.3 \\
\hline 54 & 9.9 & 4.8 & 4.3 & 9.1 & 559.47 & 10.32 & 32.6 & 5.6 \\
\hline 55 & 12.7 & 4.4 & 4.3 & 8.7 & 551.4 & 10.33 & 32 & 6.1 \\
\hline 56 & 12.9 & 6.2 & 6 & 12.2 & 479.68 & 10.35 & 32.4 & 7.4 \\
\hline 57 & 12.1 & 7.2 & 7 & 14.2 & 380.17 & 10.34 & 32.3 & 5.3 \\
\hline 58 & 11.7 & 5.1 & 4.9 & 10 & 483.26 & 10.34 & 32 & 5.5 \\
\hline 59 & 10.3 & 4.7 & 4.5 & 9.2 & 380.17 & 10.34 & 32.2 & 5.7 \\
\hline 60 & 11.3 & 8 & 4.4 & 12.4 & 497.61 & 10.34 & 32.2 & 6 \\
\hline 63 & 7.3 & 8 & 7.7 & 15.7 & 461.75 & 10.33 & 33 & 5.3 \\
\hline 64 & 6.8 & 8.2 & 8 & 16.2 & 470.71 & 10.35 & 32.1 & 6.7 \\
\hline 65 & 5.7 & 3.7 & 3.4 & 7.1 & 316.52 & 10.34 & 33 & 6.6 \\
\hline 66 & 10.1 & 5.8 & 5.6 & 11.4 & 327.28 & 10.35 & 32.8 & 6.2 \\
\hline 67 & 9 & 4.6 & 4.5 & 9.1 & 371.2 & 10.34 & 33 & 6.3 \\
\hline 68 & 12.1 & 6.5 & 5.9 & 12.4 & 370.31 & 10.34 & 32.8 & 6 \\
\hline 69 & 11.1 & 5.5 & 5.4 & 10.9 & 425.89 & 10.37 & 38 & 6.9 \\
\hline 70 & 10.7 & 6.5 & 6.2 & 12.7 & 362.24 & 10.57 & 32.8 & 7.1 \\
\hline 71 & 8.9 & 7.7 & 7.6 & 15.3 & 454.58 & 10.35 & 33 & 6.2 \\
\hline 72 & 6 & 6.2 & 5.9 & 12.1 & 465.33 & 10.33 & 32.8 & 5.9 \\
\hline 73 & 8.9 & 5.8 & 5.5 & 11.3 & 434.85 & 10.34 & 33.2 & 5.6 \\
\hline 74 & 7.6 & 8.1 & 7.9 & 16 & 424.1 & 10.35 & 33 & 6.1 \\
\hline 75 & 6.6 & 6.1 & 5.8 & 11.9 & 559.47 & 10.33 & 33 & 5.7 \\
\hline 76 & 6.5 & 7.1 & 6.9 & 14 & 360.45 & 10.33 & 32.8 & 5.6 \\
\hline 77 & 7.3 & 6.1 & 5.9 & 12 & 351.48 & 10.36 & 32.4 & 6.3 \\
\hline 78 & 6.7 & 6.9 & 6.5 & 13.4 & 481.47 & 10.34 & 32.5 & 5.8 \\
\hline 79 & 8.6 & 5.6 & 5.4 & 11 & 397.2 & 10.34 & 32.8 & 6.3 \\
\hline 80 & 10.1 & 8.3 & 8 & 16.3 & 369.41 & 10.34 & 33 & 5.9 \\
\hline 81 & 7.4 & 7.3 & 7.1 & 14.4 & 554.09 & 10.33 & 32.9 & 5.7 \\
\hline 82 & 5.8 & 5.7 & 5.5 & 11.2 & 532.57 & 10.39 & 32.8 & 7 \\
\hline
\end{tabular}


Table Continued

\begin{tabular}{|c|c|c|c|c|c|c|c|c|}
\hline $\mathbf{S} / \mathbf{N}$ & Chloride (mg/l) & Calcium (mg/l) & $\mathrm{Mg}^{2+}(\mathrm{mg} / \mathrm{l})$ & $\begin{array}{l}\text { Total } \\
\text { hardness } \\
(\mathrm{mg} / \mathrm{l})\end{array}$ & Sulphate (mg/l) & $\mathrm{PO}_{4}^{2+}(\mathrm{mg} / \mathrm{l})$ & Temp. $\left({ }^{\circ} \mathrm{C}\right)$ & pH \\
\hline 83 & 9.4 & 6.3 & 6 & 12.3 & 551.4 & 10.4 & 33.8 & 6.4 \\
\hline 84 & 8.4 & 6.8 & 6.6 & 13.4 & 541.54 & 10.36 & 33.1 & 6.7 \\
\hline 85 & 6.3 & 7.1 & 6.9 & 14 & 517.33 & 10.36 & 33 & 6.7 \\
\hline 86 & 5.2 & 6.1 & 5.8 & 11.9 & 539.74 & 10.35 & 32.6 & 6.2 \\
\hline 87 & 7.7 & 7.7 & 7.4 & 15.1 & 405.27 & 10.33 & 32.6 & 6.6 \\
\hline 88 & 6.6 & 8.6 & 8.3 & 16.9 & 554.98 & 10.39 & 32.9 & 6 \\
\hline 89 & 9.3 & 5.6 & 5.3 & 10.9 & 370.31 & 10.38 & 33.1 & 5.4 \\
\hline 90 & 5.5 & 7 & 6.7 & 13.7 & 411.55 & 10.35 & 32.5 & 5.8 \\
\hline 91 & 2.3 & 3.8 & 27.1 & 30.9 & 518.23 & 10.37 & 24.5 & 7 \\
\hline 93 & 5.7 & 4 & 5.3 & 9.3 & 860.69 & 10.45 & 25 & 6.7 \\
\hline 94 & 4.9 & 2 & 9 & 11 & 751.31 & 10.47 & 25.5 & 6.8 \\
\hline 95 & 4.9 & 2 & 9.8 & 11.8 & 827.52 & 10.34 & 26 & 7 \\
\hline 96 & 5.9 & 2 & 9.4 & 11.4 & 751.32 & 10.48 & 24.5 & 6.8 \\
\hline 97 & 8.2 & 3 & 20.1 & 23.1 & 598.02 & 10.33 & 24 & 7.2 \\
\hline 98 & 7.8 & 3.3 & 24.2 & 27.5 & 688.56 & 10.48 & 24 & 7 \\
\hline 99 & 5.6 & 3.8 & 29.9 & 33.7 & 676.01 & 10.32 & 24.5 & 7 \\
\hline 100 & 7.2 & 4 & 11.5 & 15.5 & 819.45 & 10.34 & 24 & 6.6 \\
\hline
\end{tabular}

Table 6 Physical and bacteriological quality of water samples in Ogbomoso North and South local government

\begin{tabular}{|c|c|c|c|c|c|c|}
\hline $\mathbf{S} / \mathbf{N}$ & Suspended solids $\mathrm{mg} / \mathrm{L}$ & Dissolved solids mg/L & Total solids $\mathrm{mg} / \mathrm{L}$ & E-coli mg/L & T-coli mg/L & Turbidity mg/L \\
\hline 1 & 70 & 30 & 100 & 48 & 20 & 0 \\
\hline 2 & 30 & 20 & 50 & 48 & 48 & 0 \\
\hline 3 & 62 & 40 & 102 & 44 & 44 & 0 \\
\hline 4 & 10 & 60 & 70 & 46 & 32 & 0 \\
\hline 5 & 17 & 20 & 37 & 36 & 40 & 0 \\
\hline 6 & 30 & 50 & 80 & 40 & 32 & 0 \\
\hline 7 & 40 & 80 & 120 & 24 & 40 & 0 \\
\hline 8 & 19 & 30 & 49 & 32 & 40 & 1 \\
\hline 9 & 30 & 40 & 70 & 54 & 20 & 0 \\
\hline 10 & 20 & 50 & 70 & 60 & 52 & 0 \\
\hline 11 & 30 & 30 & 60 & 20 & 32 & 0 \\
\hline 12 & 73 & 80 & 153 & 36 & 40 & 0 \\
\hline 13 & 70 & 60 & 130 & 36 & 36 & 0 \\
\hline 14 & 20 & 100 & 120 & 16 & 40 & 0 \\
\hline 15 & 110 & 90 & 200 & 48 & 41 & 0 \\
\hline 16 & 60 & 80 & 140 & 36 & 30 & 0 \\
\hline 17 & 20 & 30 & 50 & 40 & 42 & 0 \\
\hline 18 & 130 & 60 & 190 & 28 & 32 & 0 \\
\hline 19 & 40 & 60 & 100 & 42 & 30 & 0 \\
\hline 20 & 20 & 30 & 50 & 44 & 38 & 0 \\
\hline 21 & 120 & 60 & 180 & 32 & 26 & 0 \\
\hline
\end{tabular}


Table Continued

\begin{tabular}{|c|c|c|c|c|c|c|}
\hline $\mathbf{S} / \mathbf{N}$ & Suspended solids mg/L & Dissolved solids mg/L & Total solids $\mathrm{mg} / \mathrm{L}$ & E-coli mg/L & T-coli mg/L & Turbidity mg/L \\
\hline 22 & 120 & 30 & 150 & 32 & 32 & 0 \\
\hline 23 & 20 & 30 & 50 & 42 & 40 & 0 \\
\hline 24 & 110 & 40 & 150 & 34 & 44 & 0 \\
\hline 25 & 33 & 10 & 43 & 50 & 32 & 0 \\
\hline 26 & 40 & 30 & 70 & 46 & 28 & 0 \\
\hline 27 & 20 & 40 & 60 & 40 & 24 & 0 \\
\hline 28 & 22 & 20 & 42 & 48 & 30 & 0 \\
\hline 29 & 60 & 60 & 120 & 36 & 32 & 0 \\
\hline 30 & 70 & 10 & 80 & 28 & 36 & 0 \\
\hline 31 & 17 & 46 & 63 & 36 & 38 & 0 \\
\hline 32 & 81 & 40 & 121 & 42 & 40 & 0 \\
\hline 33 & 82 & 63 & 145 & 32 & 56 & 0 \\
\hline 34 & 40 & 100 & 140 & 42 & 42 & 0 \\
\hline 35 & 42 & 30 & 72 & 50 & 22 & 0 \\
\hline 36 & 48 & 120 & 168 & 62 & 46 & 0 \\
\hline 37 & 59 & 120 & 179 & 42 & 44 & 0 \\
\hline 38 & 46 & 55 & 101 & 40 & 28 & 0 \\
\hline 39 & 116 & 50 & 166 & 38 & 42 & 0 \\
\hline 40 & 75 & 49 & 124 & 34 & 48 & 0 \\
\hline 41 & 45 & 65 & 110 & 34 & 52 & 0 \\
\hline 42 & 60 & 88 & 148 & 46 & 48 & 0 \\
\hline 43 & 54 & 80 & 134 & 40 & 32 & 0 \\
\hline 44 & 43 & 73 & 116 & 26 & 38 & 0 \\
\hline 45 & 66 & 100 & 166 & 40 & 38 & 0 \\
\hline 46 & 76 & 90 & 166 & 44 & 32 & 0 \\
\hline 47 & 32 & 58 & 90 & 42 & 43 & 0 \\
\hline 48 & 26 & 60 & 86 & 36 & 48 & 0 \\
\hline 49 & 32 & 56 & 88 & 36 & 40 & 0 \\
\hline 50 & 40 & 60 & 100 & 42 & 38 & 0 \\
\hline 51 & 65 & 44 & 109 & 40 & 56 & 0 \\
\hline 52 & 60 & 60 & 120 & 40 & 44 & 0 \\
\hline 53 & 58 & 43 & 101 & 32 & 40 & 0 \\
\hline 54 & 60 & 56 & 116 & 52 & 44 & 0 \\
\hline 55 & 62 & 34 & 96 & 44 & 36 & 0 \\
\hline 56 & 58 & 40 & 98 & 32 & 40 & 0 \\
\hline 57 & 60 & 60 & 120 & 32 & 28 & 0 \\
\hline 58 & 65 & 78 & 143 & 32 & 44 & 0 \\
\hline 59 & 72 & 84 & 156 & 24 & 28 & 0 \\
\hline 60 & 68 & 32 & 100 & 52 & 68 & 0 \\
\hline 61 & 68 & 54 & 122 & 38 & 43 & 0 \\
\hline 62 & 56 & 66 & 122 & 44 & 32 & 0 \\
\hline 63 & 50 & 78 & 128 & 50 & 42 & 0 \\
\hline 64 & 70 & 36 & 106 & 48 & 36 & 0 \\
\hline
\end{tabular}

Citation: Ibironke OO,Adegoke OJ, Akindipe RD. Development of water quality map for Ogbomoso metropolis. MOJ Eco Environ Sci. 20I7;2(3): I2I-134. DOI: 10.15406/mojes.2017.02.00025 


\begin{tabular}{|c|c|c|c|c|c|c|}
\hline $\mathbf{S} / \mathbf{N}$ & Suspended solids mg/L & Dissolved solids mg/L & Total solids mg/L & E-coli mg/L & T-coli mg/L & Turbidity mg/L \\
\hline 65 & 76 & 98 & 174 & 68 & 60 & 0 \\
\hline 67 & 65 & 54 & 119 & 34 & 24 & 0 \\
\hline 68 & 60 & 52 & 112 & 44 & 36 & 0 \\
\hline 70 & 25 & 64 & 89 & 40 & 32 & 0 \\
\hline 71 & 44 & 28 & 72 & 42 & 60 & 0 \\
\hline 72 & 65 & 26 & 91 & 56 & 32 & 0 \\
\hline 73 & 66 & 42 & 108 & 46 & 20 & 0 \\
\hline 74 & 45 & 46 & 91 & 20 & 48 & 0 \\
\hline 75 & 60 & 86 & 146 & 44 & 46 & 0 \\
\hline 76 & 44 & 65 & 109 & 48 & 44 & 0 \\
\hline 77 & 70 & 2898 & 3224 & 0 & & 0 \\
\hline 78 & 26 & 50 & 76 & 40 & 52 & 0 \\
\hline 79 & 63 & 98 & 161 & 40 & 32 & 0 \\
\hline 80 & 66 & 68 & 134 & 44 & 40 & 0 \\
\hline 81 & 20 & 80100 & 4036 & & 0 & 0 \\
\hline 82 & 28 & 70 & 98 & 36 & 42 & 2 \\
\hline 83 & 21 & 70 & 91 & 46 & 44 & 1 \\
\hline 84 & 46 & 45 & 91 & 42 & 48 & 0 \\
\hline 85 & 30 & 70 & 100 & 44 & 44 & 0 \\
\hline 87 & 80 & 65 & 145 & 20 & 36 & 0 \\
\hline 88 & 80 & 50 & 130 & 64 & 40 & 0 \\
\hline 89 & 60 & 65 & 125 & 48 & 24 & 0 \\
\hline 90 & 48 & 55 & 103 & 32 & 28 & 1 \\
\hline 91 & 80 & 60 & 140 & 36 & 30 & 0 \\
\hline 92 & 15 & 60 & 75 & 23 & 27 & 0 \\
\hline 93 & 70 & 70 & 140 & 31 & 28 & 0 \\
\hline 94 & 55 & 85 & 140 & 45 & 35 & 0 \\
\hline 95 & 60 & 50 & 110 & 20 & 19 & 0 \\
\hline 96 & 100 & 35 & 135 & 26 & 30 & 0 \\
\hline 97 & 60 & 65 & 125 & 19 & 13 & 0 \\
\hline 98 & 85 & 15 & 100 & 28 & 30 & 0 \\
\hline 99 & 80 & 35 & 115 & 14 & 12 & 0 \\
\hline 100 & 20 & 30 & 50 & 16 & 18 & 0 \\
\hline
\end{tabular}

\section{Discussion}

$\mathrm{pH}$

The $\mathrm{pH}$ value of tested water samples ranged from 5.3 to 7.40. Results of tests on Samples 57, 61 and 63 taken from Akata (Abidogun 3 house), Ibapan (Ola-Oluwa Communications) and Ijeru (Bathel Baptist Church) respectively showed the least $\mathrm{pH}$ value of 5.3 indicating its acidity. $\mathrm{pH}$ values of samples $1,3,11,14,15,21,30,32,3$ $3,36,38,39,41,43,45,49,54,57,58,59,60,61,62,63,66,67,68,71,72,73$,
$74,75,76,77,78,79,80,81,83,86,88,89$ and 90 fall below the NIS and WHO standards of range 6.5-8.5. These water samples are likely to have metallic or sour taste, resulting from the leaching of metals from the surroundings to the water source.

\section{Lead $(\mathrm{Pb})$}

The Lead value ranged from $0.0 \mathrm{mg} / 1$ to $0.88 \mathrm{mg} / 1$. Samples $2,3,4$, 8,14 and 62 shows the lowest value $(0 \mathrm{mg} / \mathrm{l})$ while sample 96 shows the highest value $(0.88 \mathrm{mg} / \mathrm{l})$. All samples exceed the limit of N.I.S 
and below W.H.O standard of $0.01 \mathrm{mg} / 1$ and $1.05 \mathrm{mg} / 1$ respectively. Table 4 shows the concentration of lead in the water samples. Higher lead concentration in sample is as a result of presence of batteries, burnt tires. Research shows that people who drink water containing Lead in excess of the action level may experience delay in physical and mental development with short term exposure while in adult may cause blood pressure and adult who drink over many years could cause kidney problem.

\section{Chromium}

The Chromium value ranged from $0.048 \mathrm{mg} / 1$ to $0.199 \mathrm{mg} / 1$. Sample 91 shows the lowest value $(0.048 \mathrm{mg} / 1)$ while sample 100 shows the highest value $(0.199 \mathrm{mg} / 1)$. Samples $1,2,3$ are within the range of WHO of $0.05 \mathrm{mg} / 1$ while all samples are over NIS standard $0.1 \mathrm{mg} / 1$. Table 4 shows the concentration of Chromium in the water samples. Higher Chromium concentration in sample was a result of presence of batteries and industrial waste. Research shows that people who drink water containing Chromium in excess of the action level may with experience lung, respiratory tract cancer and kidney diseases short term exposure and with long term exposure causes diarrhea.

\section{Zinc (Zn)}

The Zinc value ranged from $0.00 \mathrm{mg} / 1$ to $0.3 \mathrm{mg} / \mathrm{l}$. Samples 30,35 , $40,54,59,67,76,84,92$ and 100 shows the lowest value $(0.00 \mathrm{mg} / \mathrm{l})$ while sample 79 shows the highest value $(0.3 \mathrm{mg} / 1)$. All samples are within the range of NIS and WHO standard of $\leq 5 \mathrm{mg} / \mathrm{l}$. Table 4 shows the concentration of Zinc in the water samples. Higher Zinc concentration in samples is as a result of presence of batteries and vehicles emission within the area. Research shows that people who drink water containing Zinc in excess of the action level may with short term exposure experience fever and intestinal disturbance.

\section{Manganese (Mn)}

The Manganese value ranged from $0.08 \mathrm{mg} / 1$ to $4.46 \mathrm{mg} / 1$. Sample 99 shows the lowest value $(0.08 \mathrm{mg} / \mathrm{l})$ while sample 33 shows the highest value $(4.46 \mathrm{mg} / \mathrm{l})$. Samples 7 and 9 are within the range of NIS and WHO standard of $0.2 \mathrm{mg} / 1$ and $0.2 \mathrm{mg} / 1$ respectively. Table 4 shows the concentration of Manganese in the water samples. Higher Manganese concentration in sample is as a result of industrial waste and vehicle emission as they are the major source of Manganese within the area. Research shows that people who drink water containing Manganese in excess of the action level may cause damage of central nervous system, weakness and fatigue.

\section{Nickel (Ni)}

The Nickel value ranged from $0.01 \mathrm{mg} / 1$ to $0.18 \mathrm{mg} / 1$. Samples 20 and 28 shows the lowest value $(0.01 \mathrm{mg} / \mathrm{l})$ while samples 55 and 77 shows the highest value $(0.18 \mathrm{mg} / \mathrm{l})$. All samples contain Nickel concentration above the range of NIS and WHO standard of $0.02 \mathrm{mg} / 1$ except samples 7, 20, 24, 28, 29, 34, 41 and 84 . Table 4 shows the concentration of nickel in the water samples. Higher Nickel concentration in sample is as a result of vehicle emission contamination as they are the major source of Nickel within the area. Research shows that people who drink water containing Nickel in excess of the action level may experience lung cancer.

\section{Cadmium (Cd)}

The Cadmium value ranged from $0.00 \mathrm{mg} / 1$ to $0.07 \mathrm{mg} / \mathrm{l}$. Samples $28,90,92,94$ to 100 shows the lowest value $(0.00 \mathrm{mg} / \mathrm{l})$ while sample 47 shows the highest value $(0.07 \mathrm{mg} / 1)$. Most samples are within the range of NIS and WHO standard of $0.03 \mathrm{mg} / 1$ and $0.05 \mathrm{mg} / 1$ respectively, only samples $31,34,38$ and 47 exceed the regulatory limits. Table 4 shows the concentration of Cadmium in the water samples. Higher Cadmium concentration in sample is as a result of presence of electroplating, batteries as they are the major source of Cadmium within the area. Research shows that people who drink water containing Cadmium in excess of the action level may experience fatigue, headache and vomiting.

\section{Iron (Fe)}

The Iron concentration value ranged from 0.0 to 0.38 .Samples $2,35,12,14,15,18-24,26-36,38-79$ and 81-90 shows the lowest value $(0.0)$ while samples 91 and 92 shows the highest value $(0.38)$. All samples are within the range WHO standard and NIS standard of $0.3 \mathrm{mg} / 1$ and $1 \mathrm{mg} / 1$ respectively. Table 4 shows the concentration of Iron in the water samples. Higher Iron concentration in sample is as a result of vehicle emission and industrial waste as they are the major source of Iron within the area. Research shows that people who drink water containing Iron in excess of the action level may experience nausea, abdominal pain and gastrointestinal bleeds.

\section{Copper (Cu)}

The Copper value ranged from $0.0 \mathrm{mg} / 1$ to $0.1 \mathrm{mg} / 1$. Samples $13-24$, 26-38, 41-56, 58-62, 64-75, 77-79 and 81-100 shows the lowest value $(0.0 \mathrm{mg} / \mathrm{l})$ while sample 3 shows the highest value of copper present. All samples are within the range of NIS and WHO standard of $1 \mathrm{mg} / 1$ and $1.5 \mathrm{mg} / \mathrm{l}$ respectively. Table 4 shows the concentration of Copper in the water samples.

\section{Temperature}

The temperature value ranged from $24^{\circ} \mathrm{C}$ to $38^{\circ} \mathrm{C}$. Samples 97 , 98 and 100 shows the lowest value $\left(24^{\circ} \mathrm{C}\right)$ while sample 71 shows the highest value $\left(38^{\circ} \mathrm{C}\right)$. All samples are within the range of NIS and WHO standard of $39.7^{\circ} \mathrm{C}$. Table 5 shows the concentration of temperature in the water samples.

\section{Chloride (Cl-)}

The Chloride value ranged from $2.2 \mathrm{mg} / 1$ to $13.2 \mathrm{mg} / 1$. Sample 3 shows the lowest value $(2.2 \mathrm{mg} / \mathrm{l})$ while sample 32 shows the highest value $(13.2 \mathrm{mg} / \mathrm{l})$. All samples are within the range of NIS and WHO standard of $100 \mathrm{mg} / 1$ and $600 \mathrm{mg} / 1$ respectively. Table 5 shows the concentration of Chloride in the water samples.

\section{Phosphate (Po4-)}

The Phosphate value ranged from $10.32 \mathrm{mg} / 1$ to $10.57 \mathrm{mg} / 1$, sample 99 shows the lowest value (10.32) while sample 70 shows the highest value (10.57). All samples are well over the range of NIS and WHO standard both of $5 \mathrm{mg} / 1$. Table 5 shows the concentration of phosphate in the water samples. Higher Phosphate concentration in sample is as a result of septic waste, fertilizer run-off and improper treatment of waste water contamination as they are the major source of Copper within the area. Research shows that people who drink water containing Phosphate in excess of the action level may experience liver or kidney damage.

\section{Sulphate (So42-)}

The Sulphate value ranged from $103.15 \mathrm{mg} / 1$ to $860.67 \mathrm{mg} / 1$. Sample 9 shows the lowest value $(103.15 \mathrm{mg} / 1)$ while sample 93 show the highest value $(860.67 \mathrm{mg} / \mathrm{l})$. Only samples $9,10,11,12,16,18,21,2$ $2,26,31,37$ and 42 are within the range of NIS and WHO standard of 
$250 \mathrm{mg} / 1$. Table 5 shows the concentration of Sulphate in the water sample. Higher Sulphate concentration in sample is as a result of movement of water through soil and formation that contain Sulphate minerals. Research shows that people who drink water containing Sulphate in excess of the action level may experience diarrhea and dehydration.

\section{Calcium hardness}

The Calcium hardness value ranged from $2.00 \mathrm{mg} / 1$ to $14.70 \mathrm{mg} / 1$ Samples 96, 97 and 98 shows the lowest value $(2.00 \mathrm{mg} / \mathrm{l})$ while sample 13 shows the highest value $(14.7 \mathrm{mg} / \mathrm{l})$. All samples are within the range of NIS and WHO standard of $30 \mathrm{mg} / \mathrm{l}$. Table 5 shows the concentration of Calcium hardness in the water samples.

\section{Magnesium hardness}

The Magnesium hardness value ranged from $2.2 \mathrm{mg} / 1$ to $29.9 \mathrm{mg} / 1$. Sample 53 shows the lowest $(2.2 \mathrm{mg} / \mathrm{l})$ while Sample 99 shows the highest value $(29.9 \mathrm{mg} / \mathrm{l})$. Some samples are within the range of NIS and WHO standard, both of $30 \mathrm{mg} / 1$. Table 5 shows the concentration of Magnesium hardness in the water samples compared with the NIS and WHO standard. Higher Magnesium concentration in sample is as a result of dissolved Magnesium and Calcium ions when getting contact with limestone and other rocks that contain Calcium compound. Research shows that hard water was not that harmful to health but can pose serious problems in industrial setting.

\section{Total Hardness}

The total hardness value ranged from $4.5 \mathrm{mg} / 1$ to $33.7 \mathrm{mg} / 1$. Sample 53 shows the lowest value $(4.5 \mathrm{mg} / 1)$ while sample 99 shows the highest value $(33.7 \mathrm{mg} / \mathrm{l})$. All samples are within the range of NIS and WHO standard of $\leq 10 \mathrm{mg} / 1$ and $\leq 50 \mathrm{mg} / 1$ respectively. Table 5 shows the concentration of total hardness in the water samples. High hardness concentration in sample is as a result of dissolved Magnesium and Calcium ions when getting contact with limestone and other rocks that contain calcium compound. Research shows that hard water was not that harmful to health but can pose serious problems in industrial settings.

\section{Suspended solids}

The suspended solid value ranged from $10 \mathrm{mg} / 1$ to $130 \mathrm{mg} / 1$. Sample 7 shows the lowest value $(10 \mathrm{mg} / 1)$ while sample 18 shows the highest value (130). All samples are within the range of NIS and WHO standard of $\leq 100 \mathrm{mg} / 1$ and $\leq 150 \mathrm{mg} / 1$ respectively. Table 6 shows the concentration of suspended solid in the water samples. Research shows that people who drink water containing suspended solid in excess of the action level may suffer from heart and kidney diseases.

\section{Dissolved solids}

The dissolved solids concentration value ranged from $10 \mathrm{mg} / 1$ to $120 \mathrm{mg} / 1$. Sample 25 shows the lowest value $(10 \mathrm{mg} / \mathrm{l})$ while sample 30 shows the highest value $(120 \mathrm{mg} / \mathrm{l})$. All samples are within the range of NIS and WHO standard of $\leq 150 \mathrm{mg} / 1$ and $\leq 500 \mathrm{mg} / 1$ respectively. Table 6 shows the concentration of dissolved solids in the water samples. Research shows that people who drink water containing dissolved solid in excess of the action level may affect person who are suffering from heart and kidney diseases.

\section{Total solids}

The Total Solid value ranged from $37 \mathrm{mg} / 1$ to $200 \mathrm{mg} / 1$. Sample 5 shows the lowest value $(37 \mathrm{mg} / \mathrm{l})$ while sample 15 shows the highest value $(200 \mathrm{mg} / \mathrm{l})$. All samples are within the range of WHO standard of $\leq 500 \mathrm{mg} / 1$ while only samples $12,15,18,21,36,37,39,45,46,59,65$ and 79 exceeds NIS standard of $\leq 150 \mathrm{mg} / 1$. Table 6 shows the concentration of Total Solid in the water samples compared with NIS and WHO standard. Research shows that people who drink water containing total solid in excess of the action level may affect person who are suffering from heart and kidney diseases.

\section{E-coli}

E-coli ranged from $14 \mathrm{mg} / 1$ to $68 \mathrm{mg} / \mathrm{l}$. Sample 99 shows the lowest concentration $(14 \mathrm{mg} / \mathrm{l})$ and sample 65 shows the highest concentration $(68 \mathrm{mg} / \mathrm{l})$. NIS and WHO does not allow any number of E- Coli as they play major role in the contribution in spreading of so many diseases. All samples failed to conform to standard. High values of $E$-coli can be attributed to improper sewage disposal measures. Table 6 shows the concentration of E-coli. Research shows that people who drink water containing $E$-coli in excess of the action level may die.

\section{T-Coliform}

T-coli ranged from $12 \mathrm{mg} / 1$ to $68 \mathrm{mg} / \mathrm{l}$. Sample 99 shows the lowest concentration $(12 \mathrm{mg} / \mathrm{l})$ and sample 60 shows the highest concentration $(68 \mathrm{mg} / \mathrm{l})$. NIS and WHO does not allow any number of T-coliform as they play major roles in the contribution in spreading of so many diseases. All samples failed to conform to standard. High values of T-coliform can be attributed to improper sewage disposal measures. Table 6 shows the concentration of T-coliform compared with NIS and WHO standard. Research shows that people who drink water containing single T-coli in excess of the action level may result to death especially for children.

\section{Conclusion and recommendations}

\section{Conclusion}

Concentration of various constituents of potable water exceeding the regulatory limits set by standard organizations; affect the use of water in some areas. This research work was channelled in this light and the following conclusions were drawn in the process: The quality of water consumed by residents of the study area has significant health implications which have been discussed comprehensively in this research work. The project serves as an insight to this informative assignment that involves water sample collection in the area, laboratory tests and analyses of these results. The quality of water is a measure of its physical, chemical and bacteriological characteristics. This project justifies the above assertion by analysing the water parameters with respect to their physical, chemical and bacteriological properties. For many aquifers within the study area, the water available for consumption satisfies the safe potable water criteria, except few exemptions identified during the course of study. Properties of tested water samples like Manganese, Nickel, Chromium, Phosphate, Sulphate, T-Coliform and E- Coliform were found to be in excess as shown in Figure 3.T-Coliform, E-Coliform concentration in the tested water samples across Ogbomoso North and South LGA were observed to be in excess. This high Total Coliform counts and $E$-Coli in water samples can be attributed to presence of septic tanks, latrines, dumpsites and indiscriminate disposal of sewages which are responsible for incidence of water borne diseases in the area (Figure $3)$. 


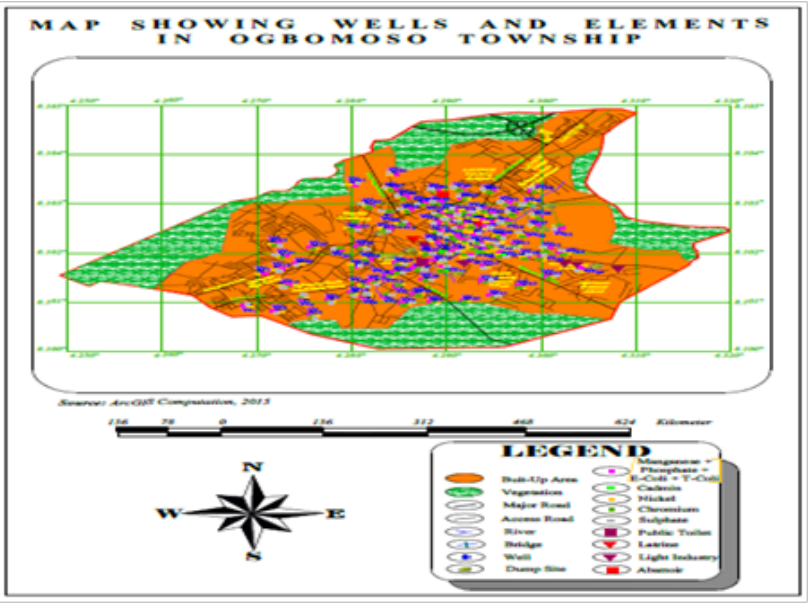

Figure 3 Water quality map for Ogbomoso North and South Local Government Area.

\section{Recommendations}

The following recommendations were made with respect to the results of analysis made on water samples in the study area:

Land use control, community mobilization, sensitization, public enlightenment, adequate collection and disposal of solid waste and prevention of grey water from bathrooms and kitchen discharge through adequate provision of drainage facilities are necessary measures to adopt towards maintaining quality potable water. Provision of well-constructed comfort stations in the area especially Agbowo and Ilogbo wards in Ogbomoso North and South LGA respectively is an urgent control measure needed. The nearness of wells to the dumpsites increases their state of pollution due to leachates migration. It is recommended that wells be dug with considerable distance from contamination sources. These wells should be properly lined and covered. It is recommended that the Nigeria Industrial Standard (NIS) saddled with the responsibility of ensuring a sustainable and healthy environment. They should develop effective measures to protect the water sources from pollution by residents, motorists and industries within the area. Government and residents of Ogbomoso South LGA especially Osupa, should work together towards making good quality water available by creating boreholes and pumps, as there are very few existing ones. Bacteriological quality of the samples in both areas is poor and unsuitable for human consumption without treatment. The bacteriological quality of the samples should be monitored by conducting a periodic on-site inspection by assessing how vulnerable the source of water is, to nearby sources of faecal contamination, testing its source-water quality periodically and disinfect water to eliminate any form of pathogens present in water.

\section{Acknowledgements}

None.

\section{Conflict of interest}

The author declares no conflict of interest.

\section{References}

1. Adriano SB, Joana AB. Original Reasearch Article. 2007;41(13):29782986.

2. United Nations development programme; 2005.

3. Sutton S. The risks of technology-based MDG indicator for rural water supply. IRC; 2008. p. 500-505.

4. Olokesusi F. Journal of Development Studies. 1990;38(5):115-120.

5. Alao AA. Evaluation of Urban water supply schemes in Oyo state. Unpublished MUPP Thesis, Centre for Urban and Regional Planning. Nigeria: University of Ibadan; 1991

6. Buckingham. World health organization and United Nations; 2000.

7. World health organization. Guidelines for Drinking Water Quality: Surveillance and Control of Communities Supplies 2nd ed. Geneva: WHO; 2000.

8. Adekunle LA, Glover RLK, Oguntola GO. Assessment of the ground water quality in Ogbomoso Township of Oyo State of Nigeria. 2011;8(1):115.

9. Ayoola PB, Adekeye EA, Jokanola OO. Environmental pollution and control within Sabo area of Ogbomoso in Oyo State of Nigeria. 2012;10(2):329.

10. Gender, Water Alliance. Mainstreaming Gender in Water Management: Resource guide, AC Dieren, Netherlands: Gender and Water Alliance; 2006.

11. Nigerian standard for drinking water quality; 2007.

12. SO Ojoawo, TL Kolade. Contamination levels of some selected Wells in Ogbomoso South local government area, Nigeria and the implications on human health. Journal of Water Resource and Protection. 2013;5(7):653668.

13. World Health Organization/United Nations International Children's Emergency Fund. Joint Monitoring Programme for Water Supply and Sanitation; 2008. 